\title{
Sexually acquired metronidazole-resistant trichomoniasis in a lesbian couple
}

\author{
D J Kellock, C P O’Mahony
}

A lesbian couple in a monogamous relationship each presented with vaginal discharge demonstrable on culture to contain Trichomonas vaginalis. Their symptoms had failed to respond to standard regimens of metronidazole, and subsequent microbiological sensitivities confirmed resistance of the trichomonads to metronidazole (minimum inhibitory concentrations in aerobic conditions $>8 \mathrm{mcg} / \mathrm{ml}$ ). In addition, the couple denied use of penetrative sex toys or recent male partners, supporting the concept of transmission through mutual masturbation. (Genitourin Med 1996;72:60-061)

Keywords: trichomonas vaginalis; lesbians; sexually transmitted diseases

\section{Introduction}

Trichomonas vaginalis (TV) is commonly found in females as a cause of vulvo-vaginitis. Regarded as a commensal when originally described by Donné in $1836,{ }^{1}$ its venereal nature was overlooked. The organism's site specificity for vaginal and urethral epithelium means that inoculation occurs primarily through sexual intercourse, with its group prevalence correlating with the level of sexual activity.

In 1989, Sivakumar et al reported the first case of sexually acquired TV in a lesbian relationship. ${ }^{2}$ This was thought to be due to the transfer of vaginal exudate on masturbating fingers, but screening of the casual partner was not possible. Some earlier studies failed to identify venereal disease in the sexually active lesbian population; however, evidence for TV infection was not always actively sought. ${ }^{3} \mathrm{~A}$ single case of trichomoniasis was also recorded in an unselected series of 27 lesbians attending a British genitourinary medicine (GUM) clinic. ${ }^{4}$ No details were given regarding the mode of transmission, although it was known that this individual had engaged in heterosexual intercourse within the last five years. A recent review of 241 self-identified lesbians attending a specialist health clinic found four cases of TV. These patients had all engaged in sexual practice with their regular partners within the last 30 days, with the timing of their most recent heterosexual contact ranging from 4 years to "never". Again, no record was made regarding sexual practice or use of sex toys (CJ Skinner, personal communication).

This case report of a metronidazole resistant trichomonal infection in a lesbian couple supports the concept of sexual transmission despite non-penetrative sexual activity and highlights the difficulty in the eradication of resistant trichomoniasis.

\section{Case one}

A 30 year old lesbian was referred with a 12 month history of green offensive vaginal discharge and pruritus vulvae. She had attended her general practitioner on four previous occa- sions, where she had received oral metronidazole therapy for TV and mixed anaerobic infection. Her symptoms had recurred on each occasion upon stopping the antibiotics. Three months prior to her presentation to ourselves, she had attended another GUM clinic and again received oral metronidazole for TV and Gardnerella vaginalis. She was in a stable two year relationship with Case Two, who had also received oral metronidazole therapy. The patient's only male contact had followed a sexual assault ten years previously.

On examination, the vulva was normal. A profuse green vaginal discharge was noted. Immediate microscopy of the discharge demonstrated TV and numerous pus cells. The provisional diagnosis was of metronidazole-resistant trichomoniasis. Serology for syphilis and hepatitis B, and cultures for gonorrhoea and chlamydia were all negative. The patient was treated with an oral dose of tinidazole 2 gms stat, repeated three days later.

At the one week review, her symptoms had eased, but a yellow discharge was now present. Examination of this demonstrated no TV; however, Gardnerella vaginalis was cultured. She was treated with clindamycin $2 \%$ vaginal cream for seven nights. Two weeks later the green discharge had returned, and TV was again identified in the wet preparation. On this occasion, her partner also attended the clinic and each received oral tinidazole 2 gms stat, repeated three days later. They were requested to abstain from further sexual practice for the duration of treatment. Over the following six weeks, Case One received several regimens in an attempt to eradicate her TV: metronidazole suppositories $500 \mathrm{mgms}$ PV nocte for three days (remained TV positive), metronidazole suppositories $1 \mathrm{gm}$ PR nocte for ten days (remained TV positive), metronidazole suppositories $1 \mathrm{gm}$ PV TDS plus $1 \mathrm{gm}$ PR TDS for ten days (TV negative). The couple were also advised to disinfect their taps and toilet seat daily, and to use separate towels to avoid non-venereal transmission from objects contaminated with body fluids.

The symptoms of odour, discharge and irritation only cleared upon completion of the 
MICs for control/case trichomonads

\begin{tabular}{llc}
\hline Control & MIC aerobic & $2 \mathrm{mcg} / \mathrm{ml}$ \\
& MIC anaerobic & 1 \\
Case one & MIC aerobic & $250 \mathrm{mcg} / \mathrm{ml}$ resistant \\
& MIC anaerobic & 2 \\
Case two & MIC aerobic & $16 \mathrm{mcg} / \mathrm{ml} \mathrm{resistant}$ \\
& MIC anaerobic & 2 \\
\hline
\end{tabular}

final regimen. Wet preparation finally demonstrated no evidence of TV, gardnerella or candida. She remained well at follow-up and was discharged from clinic.

\section{Case two}

A 33 year old lesbian attended with a four month history of clear vaginal discharge; worse in the mornings and occasionally heavy. There was no associated discomfort, pruritus or smell. She had received a week's course of oral metronidazole therapy from her general practitioner one month earlier. She was in a stable relationship with Case One. They practised mutual masturbation and had oro-genital sexual contact, but denied the use of any penetrative sex toys or vibrators. Although there had been previous male sexual partners, none of these had been in the last ten years.

On examination there was a thick white vaginal discharge. Immediate wet preparation demonstrated TV. Serology for syphilis and hepatitis B, and cultures for gonorrhoea and chlamydia were all negative. The patient, and her partner, were each treated with oral tinidazole 2 gms stat, repeated three days later.

At the one week review, further wet preparation demonstrated persisting TV, so metronidazole suppositories 500 mgms PV nocte for three days were prescribed for both patients. One week later, trichomonads were not found on the vaginal swab from Case Two. However, since her partner's TV persisted, Case Two was treated epidemiologically with rectal metronidazole $1 \mathrm{gm}$ nocte for seven days. She remained well, with no TV on wet preparation at follow up.

\section{Results}

Vaginal exudate from each patient was collected in Feinburg's medium and forwarded to Newcastle Public Health Laboratory for culture and minimum inhibitory concentrations (MIC) to metronidazole. The MIC of metronidazole under anaerobic conditions for reference strains and resistant strains are similar, ${ }^{5}$ therefore the MIC values in aerobic condition correlate better with the degree of clinical resistance. An MIC in aerobic conditions of more than $8 \mathrm{mcg} / \mathrm{ml}$ indicates "true resistance", 5 as demonstrated for trichomonads of both our patients (table).

\section{Discussion}

Metronidazole has been the drug of choice in trichomonal vaginitis for more than thirty years. Since the infection is often multifocal (vagina, Skene glands, urethra) systemic therapy is required. Treatment failure in TV infection was first described by Robinson in $1962 . .^{6}$ In those cases of treatment failure it is important to exclude non-compliance, lack of drug absorption, increased hepatic inactivation (due to interaction with other drugs), inactivation of the drug by intestinal/vaginal flora, reinfection by new or untreated partners, low serum zinc levels, or infection with resistant strains.

Resistance is not an all-or-nothing phenomenon; it is relative. Cures are therefore obtained in most cases by increased dosage or by repeated high-dose regimens. A cure was obtained in our symptomatic patient with a regimen of metronidazole suppositories $1 \mathrm{gm}$ PV tds, plus $1 \mathrm{gm}$ PR tds for ten days (a total of 60 gms of drug). This is slightly greater than the highest dose regimen reported by the British Co-operative Clinical Group in their review of recalcitrant vaginal trichomoniasis.

Our couple were well motivated, in a stable relationship, and underwent concomitant treatment, which we felt negated the variables of compliance and re-infection. In addition, the in vitro MIC values of the cultured TV were highly suggestive of resistance, supporting the impression of female to female transmission of TV through mutual masturbation.

1 Kampmeier RH. Description of Trichomonas vaginalis by MA Donné. Sex Transm Dis 1978;5:119.

2 Sivakumar K, De Silva AH, Basu Roy R. Trichomonas vaginalis infection in a lesbian. Genitourin Med 1989;65: 399-400

3 Robertson P, Schachter J. Failure to identify venereal disease in a lesbian population. Sex Transm Dis 1981;8 ease in 6 .

4 Edwards A, Nicol Thin $R$ Sexually transmitted diseases in lesbians. Int $\mathcal{F}$ STD AIDS 1990;1:178-81.

5 Ahmed-Jushuf IH, Murray AE, McKeown J. Managing trichomonal vaginitis refractory to conventional treatmen with metronidazole. Genitourin Med 1988;64:25-9.

6 Robinson SC. Trichomonal vaginitis resistant to metronidazole. Can Med Assoc F 1962;86:665.

7 British Co-operative Clinical Group. An investigation, by questionnaire, of cases of recalcitrant vaginal trichomoniasis seen in genitourinary medical clinics in the United Kingdom. Int f STD AIDS 1992;3:24-7. 